\title{
Critical Density Interaction Studies
}

Peter Young, Hector Baldis, Peter Cheung, Wojciech Rozmus, William Kruer, Scott Wilks, Steven Crowley, Warren Mori and Charles Hansen

February 14, 2001

U.S. Department of Energy

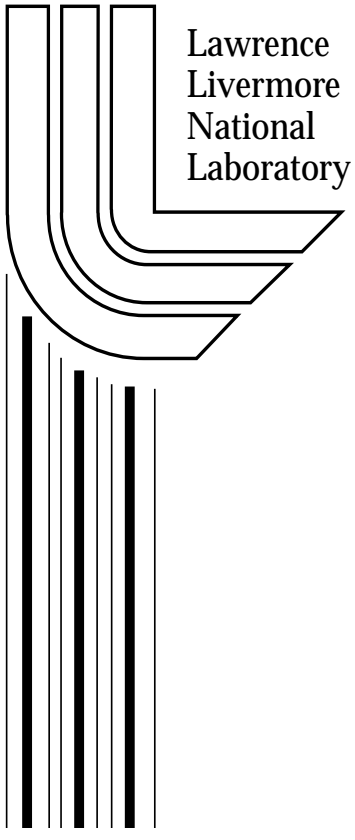




\section{DISCLAIMER}

This document was prepared as an account of work sponsored by an agency of the United States Government. Neither the United States Government nor the University of California nor any of their employees, makes any warranty, express or implied, or assumes any legal liability or responsibility for the accuracy, completeness, or usefulness of any information, apparatus, product, or process disclosed, or represents that its use would not infringe privately owned rights. Reference herein to any specific commercial product, process, or service by trade name, trademark, manufacturer, or otherwise, does not necessarily constitute or imply its endorsement, recommendation, or favoring by the United States Government or the University of California. The views and opinions of authors expressed herein do not necessarily state or reflect those of the United States Government or the University of California, and shall not be used for advertising or product endorsement purposes.

This work was performed under the auspices of the U. S. Department of Energy by the University of California, Lawrence Livermore National Laboratory under Contract No. W-7405-Eng-48.

This report has been reproduced directly from the best available copy.

Available electronically at http://www.doc.gov/bridge

Available for a processing fee to U.S. Department of Energy

And its contractors in paper from

U.S. Department of Energy

Office of Scientific and Technical Information

P.O. Box 62

Oak Ridge, TN 37831-0062

Telephone: (865) 576-8401

Facsimile: (865) 576-5728

E-mail: reports@adonis.osti.gov

Available for the sale to the public from

U.S. Department of Commerce

National Technical Information Service

5285 Port Royal Road

Springfield, VA 22161

Telephone: (800) 553-6847

Facsimile: (703) 605-6900

E-mail: orders@ntis.fedworld.gov

Online ordering: http://www.ntis.gov/ordering.htm

OR

Lawrence Livermore National Laboratory

Technical Information Department's Digital Library

http:/ / www.llnl.gov/tid/Library.html 


\title{
LDRD FINAL REPORT
}

99-ERI-007

\section{CRITICAL DENSITY INTERACTION STUDIES}

$\begin{array}{lll}\text { Principal Investigator: } & \text { Peter Young } & \text { ILSA-P\&ST } \\ \text { Co-investigators: } & \text { Hector A. Baldis } & \text { ILSA - LLNL } \\ & \begin{array}{l}\text { Peter Cheung } \\ \text { Wojciech Rozmus }\end{array} & \text { UCLA - Physics } \\ & \text { William Kruer } & \text { University of Alberta } \\ & \text { Scott Wilks } & \text { D\&NT } \\ & \text { Steven Crowley } & \text { UCLA - Physics } \\ \text { Collaborators: } & \text { Warren Mori } & \text { UCLA - Physics } \\ & & \\ \text { Postdocs: } & \text { Charles Hansen } & \text { ILSA PostDoc }\end{array}$

\begin{abstract}
Experiments have been performed to study the propagation of intense laser pulses to high plasma densities. The issue of self-focusing and filamentation of the laser pulse as well as developing predictive capability of absorption processes and x-ray conversion efficiencies is important for numerous programs at the Laboratory, particularly Laser Program (Fast Ignitor and direct-drive ICF) and D\&NT (radiography, high energy backlighters and laser cutting). Processes such as resonance absorption, profile modification, linear mode conversion, filamentation and stimulated Brillouin scattering can occur near the critical density and can have important effects on the coupling of laser light to solid targets. A combination of experiments have been used to study the propagation of laser light to high plasma densities and the interaction physics of intense laser pulses with solid targets. Nonparaxial fluid codes to study nonstationary behavior of filamentation and stimulated Brillouin scattering at high densities have also been developed as part of this project.
\end{abstract}

\section{Introduction}


For many years, the interaction of high-intensity electromagnetic (EM) waves with the coronal plasma surrounding ignition targets has been of interest to numerous efforts at the Laboratory, including the Fast Ignitor project, direct-drive studies for the National Ignition Facility (NIF), and studies involving $\mathrm{x}$-ray radiography research, high-energy $\mathrm{x}$-ray backlighters, and laser cutting. Understanding laser propagation through underdense plasmas is important because in many laser-target experiments, the laser beam can filament or self-focus - this can change the laser intensity at the target from what one would measure in a vacuum. A secondary effect of filamentation is that the levels of the stimulated Brillouin and Raman scattering instabilities (SBS and SRS, respectively) can change because filamentation changes the laser intensity, the density, and the density gradients on which the growth of SBS and SRS depend. These instabilities may be important because SBS can also modify the amount of light coupling into the target, and SRS can generate hot electrons that can heat the target.

In this project we addressed three aspects of laser-target coupling to plasma densities near the critical density, $\mathrm{n}_{\mathrm{c}}$, where the plasma frequency equals the laser frequency: (1) absorption mechanisms in high intensity, short pulse laser-target interactions with solid targets, (2) development of simulations to accurately model the propagation of high intensity laser pulses through high density plasmas, and (3) control of laser propagation properties in underdense plasmas by changing the laser pulse shape.

In FY00, we published, in Physical Review Letters [1], the results of critical density studies using 130-fs long laser pulses at intensities of up to $5 \times 10^{16}$ $\mathrm{W} / \mathrm{cm}^{2}$. By observing the intensity scaling of the second harmonic spectral shape for different incident laser polarizations, we were able to show that the dominant absorption mechanism for near-normal incidence is the anomalous skin effect. This is an important result for understanding the coupling of high intensity light to high density plasmas.

Our collaborators at the University of Alberta developed theoretical tools to model filamentation and SBS at high plasma densities. These instabilities involve plasma waves of wavenumber $\mathrm{k}$ such that $\mathrm{k} \lambda_{\mathrm{ei}} \sim \mathrm{O}(1)$, where $\lambda_{\mathrm{ei}}$ is the mean free path for electron-ion collisions. A theory of SBS and filamentation has been derived from a nonlocal hydrodynamic model which is valid in the entire range of $\mathrm{k} \lambda_{\mathrm{ei}}$ and includes thermal processes. The results have been published in Physics of Plasmas [2]. 
We also analyzed data from the Janus laser in which a 1-ns-long laser pulse propagated through an underdense plasma. It was observed that less spreading due to the filamentation instability was observed when a Gaussian-shaped pulse was used than when a square pulse with a leading edge rise-time of 100 ps was used. Hydrodynamic simulations using adiabatically-rising pulse shapes showed that if the rise time of the pulse is slower than the time for the plasma to move out of the beam, then filamentation can be avoided if the laser channel density is lowered such that the pulse is always below the filamentation threshold. These results are being prepared for submission to Physics of Plasmas.

In the remainder of this report, we discuss each of these activities in more detail. This work has helped us to improve our understanding of how to efficiently couple laser energy to high plasma densities.

\section{High-intensity interactions with solid targets}

The study of laser-plasma interactions has long been concerned with the process of laser light absorption. Resonance absorption [3] at the critical density often makes a significant contribution to the net absorption and observations of second harmonic $(\mathrm{SH})$ emission produced by resonance absorption were used in early nanosecond pulse length laser experiments to understand this phenomenon [4]. With the recent advent of ultra-short pulse, high-intensity lasers, the absorption of light near the critical density again becomes an important subject of investigation and $\mathrm{SH}$ emission again can be used as a diagnostic of densities near $n_{c}[5,6]$, where $n_{c}$ is the critical density at laser wavelength, $\lambda$. Very short density gradient scalelengths, $L / \lambda<<1$ are possible because the laser-produced plasma does not have time to expand significantly during the laser pulse. Under these conditions, the interaction takes place in the skin layer of an overdense plasma $\left(n_{e} \gg n_{c}\right)$. At laser intensities below $10^{16} \mathrm{~W} / \mathrm{cm}^{2}$, electron temperatures are in the sub-keV range and the main laser absorption mechanism is related to electron collisions in the skin layer. At higher laser intensities, the electron mean free path exceeds the skin depth leading to collisionless energy absorption $[7,8]$. Several collisionless mechanisms have been proposed, including vacuum heating, $\mathrm{J} \times \mathrm{B}$ heating, and the anomalous skin effect [9].

In this section, we describe blue shifts of up to $70 \AA$ in the SH generated by the irradiation of a solid target with a 130-fs laser pulse for p-polarization, 
an incident angle of $17.5^{\circ}$, and laser intensities above $5 \times 10^{16} \mathrm{~W} / \mathrm{cm}^{2}$. The intensity at which the shift first appears is consistent with the predicted onset of the anomalous skin effect. We show for the first time that the $\mathrm{SH}$ spectrum can be calculated from the nonlinear electron orbits that result when the laser intensity is large enough to drive the electrons into the target a distance greaater than the skin depth, and the effect is also observed in collisionless PIC simulations. For a large angle of incidence, near $55^{\circ}$, the second harmonic remains unshifted even at the highest intensities and the behavior is consistent with resonance absorption. Previously reported experiments that have observed $\mathrm{SH}$ have either been at much lower intensities [5] or not spectrally resolved [6].

The experiments were performed with the USP laser at Lawrence Livermore National Laboratory. The laser produces a 130 -fs pulse at a peak wavelength of $800 \mathrm{~nm}$. In the present experiment, energies ranged from $120 \mathrm{~mJ}$ down to a few $\mu \mathrm{J}$. The optical compression of the laser pulse and the propagation to the target took place in vacuum. The contrast ratio [10] of the $800 \mathrm{~nm}$ peak to $1 \mathrm{ps}$ earlier or later is $10^{-5}$ and the power ratio of the compressed pulse to the ASE level is $10^{-7}$. Independent measurements of the density gradient scale lengths close to the critical density show that they are shorter than the laser wavelength.

The incident laser light was monitored with a spectrometer; this is important for analyzing the scattered light spectrum because the laser spectrum and central frequency can change from day to day. Approximately $0.5 \%$ of the incident laser light is allowed to leak through a turning mirror to record the incident spectrum on each shot. The incident light spectrometer is calibrated to $\pm 0.12 \mathrm{~nm}$.

The pulse was focused onto the surface of a solid target with an $f$ /3.6 offaxis parabolic mirror at several angles of incidence relative to target normal with either s- or p-polarization selected using a rotating reflector system. This produced an ellipsoidal spot of approximately $7 \mu \mathrm{m}$ by $25 \mu \mathrm{m}$ containing $50 \%$ of the incident energy; intensities of up to $4 \times 10^{17} \mathrm{~W} / \mathrm{cm}^{2}$ were obtained. The target was an $0.5 \mu \mathrm{m}$ layer of aluminum deposited on an optically flat glass substrate. The target was rastered between shots to ensure that the laser interacted with an undamaged surface, and was positioned in the focal direction to ensure that the spot size remained constant throughout the experiment. 
The light from the target in the specular direction was collected with an $f / 6$ spherical mirror which imaged the interaction region onto the slit of an optical spectrometer. This spectrometer has a wavelength accuracy and resolution similar to the incident light spectrometer $( \pm 0.12 \mathrm{~nm}$.)

The near-field pattern of the specularly-reflected SH light was obtained by placing a scatter plate $15 \mathrm{~cm}$ away from the target. The scatter plate was imaged onto a television camera and images were acquired during the shot with a frame grabber. Images of both $800 \mathrm{~nm}$ and $400 \mathrm{~nm}$ light were obtained on separate shots.

The spectral and spatial analysis of SH can be a tool towards understanding the interaction of the incident laser with its critical density. If resonance absorption is dominant, then the SH has specific signatures: since $\mathrm{SH}$ is generated by an electron plasma wave at the critical layer, its frequency will be exactly twice that of the incident laser (except for Doppler motion of the critical layer) and it will be emitted from the target primarily in the specular direction for the incident laser. Dramatic changes in the SH frequency and its direction of emittance would be signatures of non-resonant absorption processes.

Absorption processes are generally dependent on the density gradient scale length. We can verify that this scale length is much smaller than the wavelength of the incident laser in two ways. Figure 1 shows the measured specular reflection as a function of incident angle compared with theoretical predictions. As is well known $[11,12]$, the angle of peak absorption depends on the ratio $L / \lambda$ where $L$ is the density scale length at the critical density, and $\lambda$ is the incident laser wavelength. In addition to angular dependent absorption measurements, we can also measure the Doppler shift in the reflected $800 \mathrm{~nm}$ light, and infer a density gradient scale length. The 


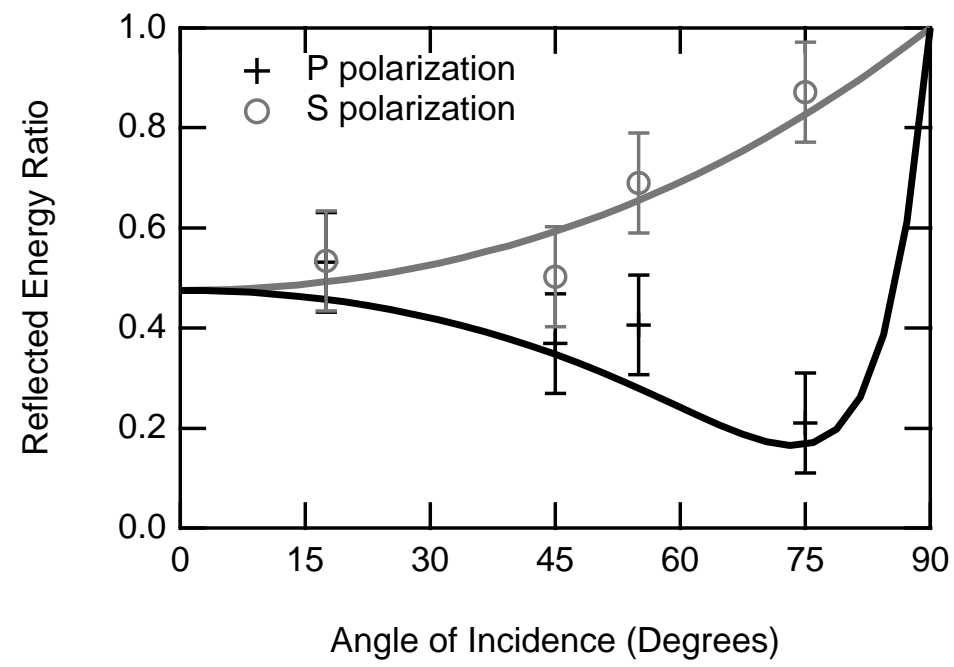

Figure 1. Angular dependence of the target reflectivity for an incident intensity of $\sim 3 \mathrm{x}$ $10^{16} \mathrm{~W} / \mathrm{cm}^{2}$. The solid lines are calculations based on the references in the text with $\mathrm{L} / \lambda$ $\sim 0.3$. fundamental is blue-shifted $\sim 1.7 \mathrm{~nm}$ corresponding to an expansion velocity $\mathrm{c}_{\mathrm{s}} / \mathrm{c} \sim 2 \times 10^{-3}$ which is consistent with earlier results [12,13] and with the density gradient scale length inferred from Figure 1.

In the present experiment, we observe behavior both consistent and inconsistent with the resonant absorption process, depending on the angle of incidence. Figure 2 summarizes the results obtained with an angle of incidence of 55 degrees. The results are consistent with resonance absorption: (1) the SH light is confined to the specular direction (see Fig. 2a) for p-polarization and the SH signal is reduced a factor of 10 for spolarization, (2) the reflectivity is roughly independent of intensity and has the expected dependence on polarization (see Fig. 2b), and (3) the frequency of the $\mathrm{SH}$ emission is very close to twice the incident laser frequency (see Fig. 2c).

Figure 3 shows the same set of data as Figure 2, but at an incident angle of $17.5^{\circ}$ with respect to the target normal. This differs from the larger angle of incidence data in three main ways. The first is that the SH light is no longer primarily emitted in the specular direction, even though the fundamental still is (see Fig. 3a). This indicates that the $\mathrm{SH}$ due to electron plasma waves at the $\mathrm{n}_{\mathrm{c}}$ surface are dominated by another $\mathrm{SH}$ production process. The second difference is that the fraction of energy reflected is strongly dependent on incident intensity. The third is that the $\mathrm{SH}$ spectrum is not always centered precisely at twice the incident laser frequency. 

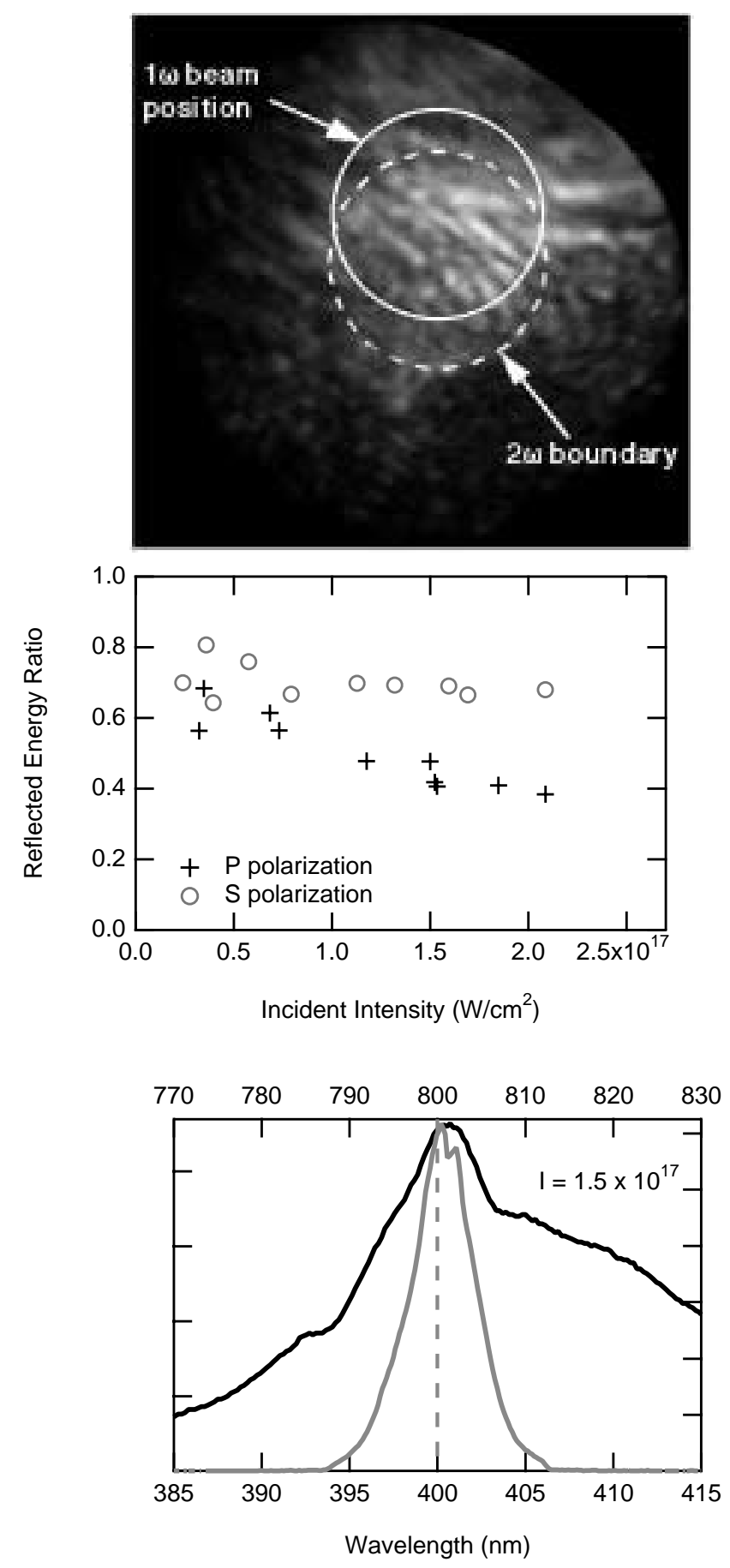

Figure 2. Summary of the second harmonic at an angle of incidence of 55 degrees. (a) An image of the reflected SH light in the far field, $2.1 \times 10^{17} \mathrm{~W} / \mathrm{cm}^{2}$. The circle represents the location of the specular light cone. (b) Total energy in the specular direction as a function of incident intensity. (c) SH spectrum in the specular direction (black) and the incident laser spectrum (gray). 


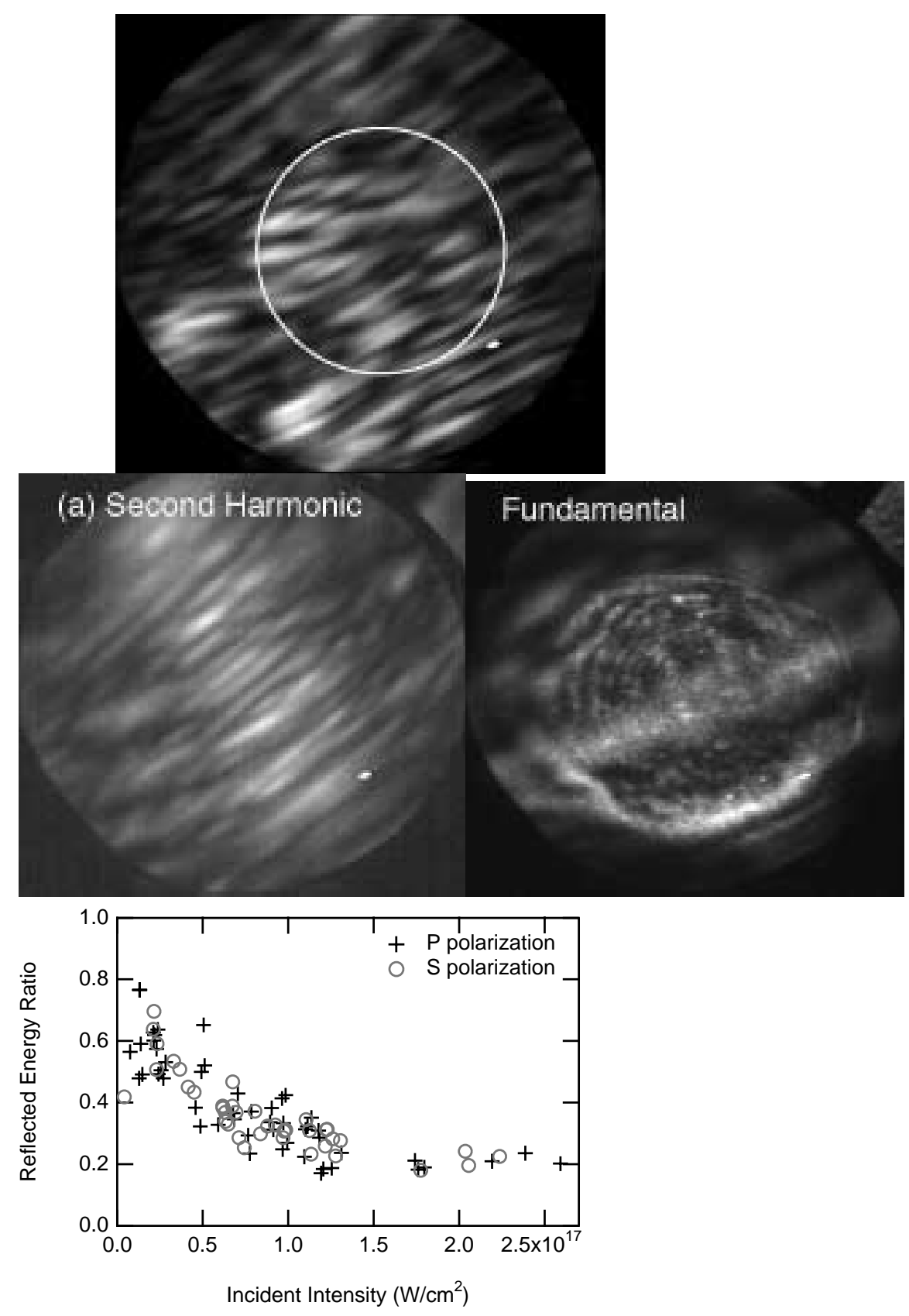




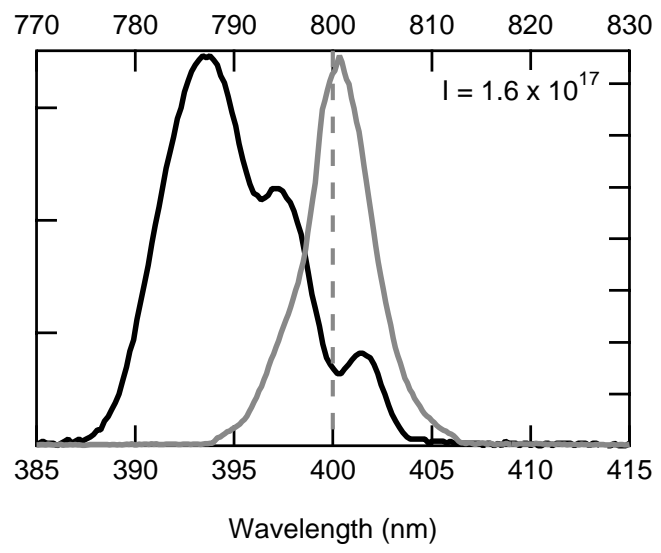

Figure 3. Summary of the second harmonic at an angle of incidence of 17.5 degrees. (a) Images of the second harmonic and the fundamental reflected light in the far field, for 9 $\mathrm{x} 10^{16} \mathrm{~W} / \mathrm{cm}^{2}$. (b) Total energy in the specular direction as a function of incident intensity. (c) $\mathrm{SH}$ in the specular direction (black) and the incident laser spectrum (gray).

The dependence of the wavelength shift on incident laser intensity is plotted in Fig. 4; the SH peak is blue-shifted for $I_{L}>5 \times 10^{16} \mathrm{~W} / \mathrm{cm}^{2}$. This shift is much larger in magnitude than the Doppler shift seen in the spectrum of the fundamental, and therefore cannot be explained by the hydrodynamic motion of the plasma. The lowest intensity where the SH shift occurs is approximately the calculated threshold intensity for anomalous skin effect absorption $[7,8,14]$ which does not require collisions. The same calculations, as well as our Doppler measurements of the $800 \mathrm{~nm}$ light, indicate that the SH shift occurs at temperatures $\left(T_{e} \sim 800 \mathrm{eV}\right)$ high enough to significantly reduce the importance of collisions on the plasma behavior.

We have been able to observe a blue shift in the SH in PIC simulations using a fully 2-D version of the code Zohar [15] which has been modified to simulate oblique incidence. Simulations were performed with p-polarized laser pulses of length $40 \mathrm{fs}$ (FWHM), incident on an $8 \mathrm{n}_{\mathrm{c}}$ density plasma at an angle of 20 degrees. The simulation used $\mathrm{T}_{\mathrm{e}}=800 \mathrm{eV}$ and had no collisions. The ions were immobile in order to isolate the shift produced by the nonlinear electron motion from ponderomotive motion of the critical 


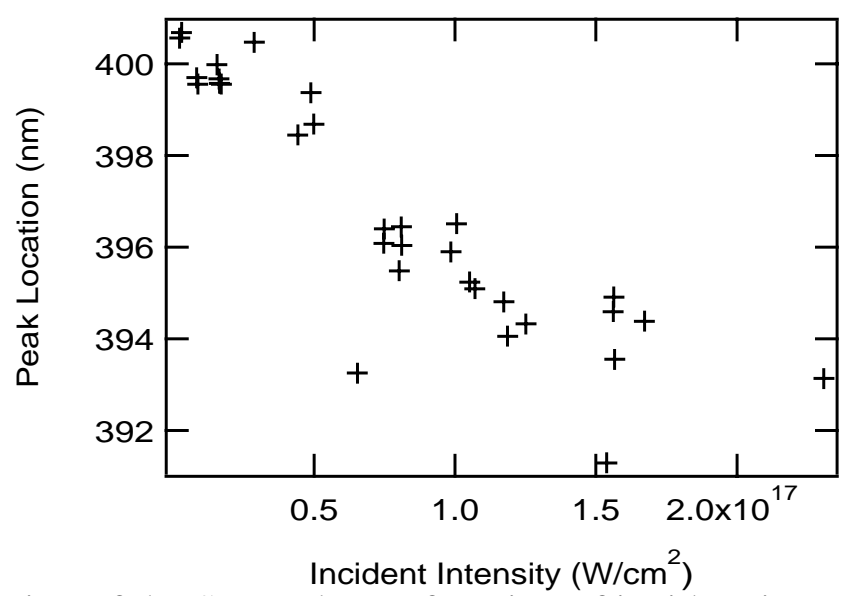

Figure 4. Location of the SH peak as a function of incident intensity at an angle of incidence of 17.5 degrees. PIC simulation results are also shown for comparison.

surface. The result of a run with an incident intensity of $1.3 \times 10^{17} \mathrm{~W} / \mathrm{cm}^{2}$, is shown in Fig. 5 which shows a blue shift and an asymmetry similar to that observed in the experiment. The difference in the magnitude of the shift between simulation and experiment could be due to laser filamentation or deformation of the critical surface, evidence of which is observed in the nonuniformities that appear in the specularly reflected $800 \mathrm{~nm}$ images at these intensities. From these results, we infer that the SH frequency shift is due to a collisionless mechanism that is intensity dependent.

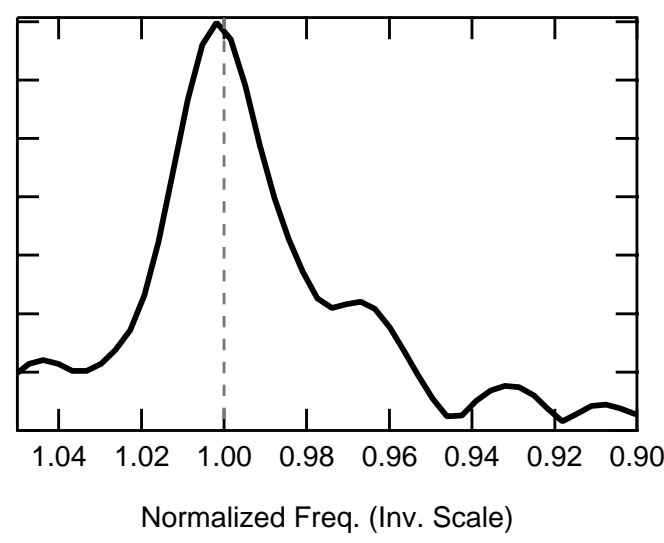

Figure 5. Frequency spectra near second harmonic, obtained from 2-D PIC simulations at an incident intensity of $1.3 \times 10^{17} \mathrm{~W} / \mathrm{cm}^{2}$.

When collisional and resonance absorption are no longer important, the electrons can be driven into the target a distance greater than the skin depth and collisionless absorption will dominate the absorption process [14,16]. If the oscillation in the sheath is nonlinear, in particular if the frequency of the 
oscillation changes with its amplitude, then the applied electromagnetic field will drive the oscillation out of resonance. This can change the frequency of the radiation emitted by the moving charges involved. To illustrate this, we consider the following Hamiltonian,

$$
H=\omega_{0} I+\alpha I^{2}+\beta \sqrt{ } I \cos \theta \cos \left(\omega_{0} t\right)
$$

where I is the action variable of the oscillation, which is proportional to the square of the amplitude, and $\theta$ is the phase, or angle variable. The parameters $\alpha$ and $\beta$ are the strengths of the nonlinearity and the drive respectively. By expanding this Hamiltonian to include $\mathrm{t}$ and $\mathrm{H}$ as canonical variables, then removing the resonance and averaging over fast time scales, a simple system can be obtained which relates the amplitude and phase modulation of the driven wave. This system can be integrated numerically. The phase and amplitude modulations are shown in the inset to Fig. 6. Note that the amplitude is largest when the phase is increasing. Therefore if the phase change is averaged over a cycle, weighted by amplitude, there will be a net increase in frequency. The radiation from these moving charges will therefore be shifted more towards the blue than if the pump is weak, or if dissipation (due to collisions) prevents the orbital amplitude from growing sufficiently to cause the motion to be nonlinear.

The Fourier transform of an oscillating system modulated by the calculated phase and amplitude is shown in Fig. 6. We see that the inclusion of a phase modulation causes the largest lobe of the Fourier transform to be shifted away from the nominal frequency. The sign of the nonlinearity $\alpha$ in Eq. 1 has been chosen for the case where the electron moves to densities above $n_{c}$, consistent with our model. This type of interaction could explain the frequency shift seen in our data and in the PIC simulations. 


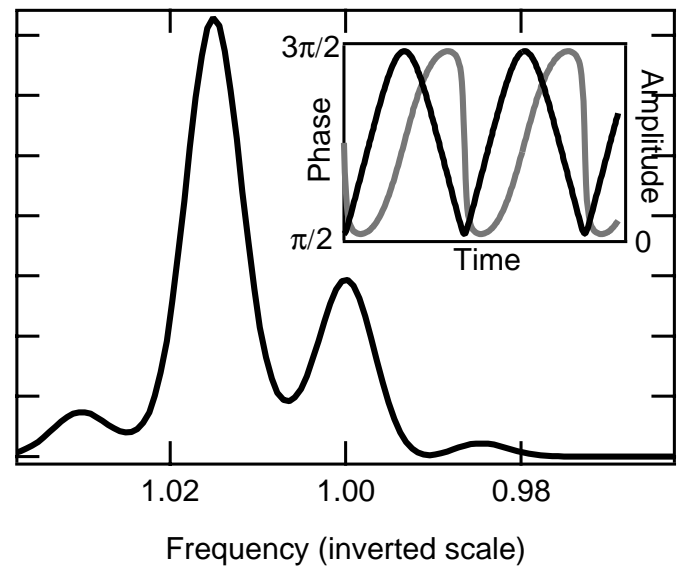

Figure 6. Magnitude squared of the Fourier transform of the modulated wave. The inset shows the phase and amplitude modulation resulting from the driven non-linear oscillator Hamiltonian (Eq. 1). The gray trace is phase while the black is amplitude.

These experiments have confirmed that absorption at higher intensities and smaller angles of incidence begins to be dominated by collisionless processes. We also see that at large angles of incidence and at intensities below $5 \times 10^{16} \mathrm{~W} / \mathrm{cm}^{2}$, resonance absorption and collisions, respectively, dominate the absorption process. This is in agreement with theory and with previous experimental results.

\section{Hydrodynamic modeling of SBS and filamentation $\mathbf{n}_{\mathrm{e}} \sim \mathbf{n}_{\mathrm{c}}$}

The main characteristic of laser plasma interaction experiments at densities close to critical density and at moderate electron temperatures, $\mathrm{T}_{\mathrm{e}}<1 \mathrm{keV}$ is the importance of thermal effects in addition to the usual ponderomotive coupling. In addition, light at higher densities is more effectively coupled to the plasma and more likely to undergo self-focusing instability. Three specific research projects have been investigated:

\section{III.A. Theory of filamentation instability and stimulated Brillouin scattering with nonlocal hydrodynamics.}

The nonlocal transport theory [18] of a laser heated plasma has been applied to analyze stimulated Brillouin scattering (SBS) and filamentation instability (FI). We have formulated a complete linear theory of SBS and FI in the full range of plasma collisionality with a proper treatment of the ponderomotive and inverse bremsstrahlung heating effects. 
As an illustration of our results we present the stationary SBS gain coefficient

$\mathrm{G}_{\mathrm{SBS}}=\left(\mathrm{A}_{2 \mathrm{k}_{-} 0} / 2\right)\left(\mathrm{c}_{\mathrm{s}} / \gamma_{\mathrm{s}}\right)\left(\omega_{\mathrm{pe}}{ }^{2} / \mathrm{c}^{2}\right)\left(\mathrm{I}_{0} / \mathrm{n}_{\mathrm{c}} \mathrm{T}_{\mathrm{e}}\right)$

where $\gamma_{\mathrm{s}}$ describes damping of ion acoustic waves and includes effects of the nonlocal electron transport, and the coefficient $\mathrm{A}_{2 \mathrm{k}_{0} 0}$ describes thermal and ponderomotive coupling. In the ponderomotive collisionless regime $\mathrm{A}_{2 \mathrm{k}_{-} 0}=$ $1 / 2$ and the deviation from this value due to thermal effects is illustrated in Fig. 7. Even in the weakly collisional plasmas, i.e. for $0.1<\mathrm{k} \lambda_{\text {ei }}<10$, the coupling coefficient is dramatically different from 0.5 value. Big dots in Fig. 7 correspond to $\mathrm{Z}=64$ and the small dots describe $\mathrm{Z}=8$ plasmas. Similar corrections are present in the expression for the filamentation gain coefficient,

$\mathrm{G}_{\mathrm{FI}}=\left(\mathrm{k} / 2\left(1-\mathrm{n}_{\mathrm{e}} / \mathrm{n}_{\mathrm{c}}\right)^{1 / 2}\left\{2\left(\mathrm{n}_{\mathrm{e}} / \mathrm{n}_{\mathrm{c}}\right)\left(\mathrm{I}_{0} / \mathrm{n}_{\mathrm{c}} \mathrm{T}_{\mathrm{e}}\right) \mathrm{A}_{\mathrm{k}}-\left(\mathrm{k}^{2} \mathrm{c}^{2} / \omega_{0}{ }^{2}\right)\right\}^{1 / 2}\right.$

where $A_{k}$ is evaluated at the characteristic wavenumber $k$.

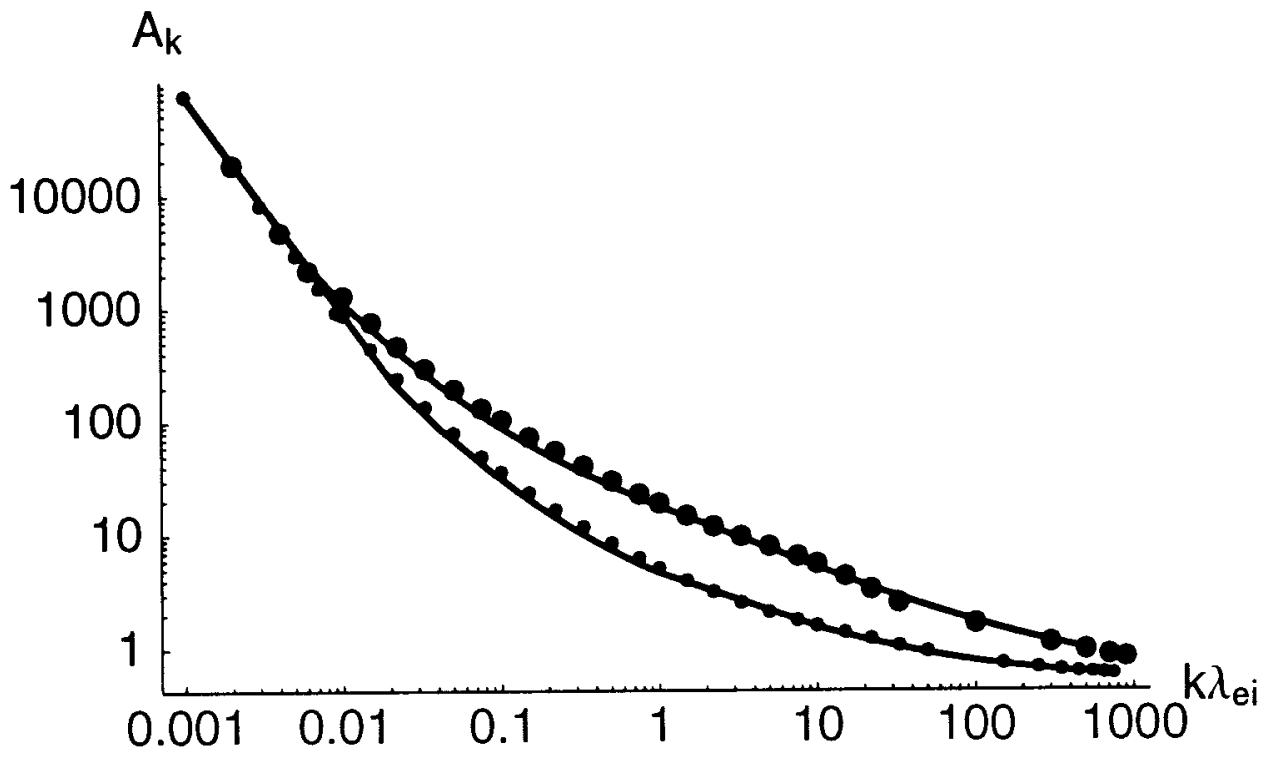


Fig 7. Coupling parameter $A_{k}$ as a function of the wavenumber $k$ normalized by the electron-ion mean-free-path, $\lambda_{\text {ei }}$

\section{III.B.Resonant instability of laser filaments in a plasma.}

Different beam smoothing techniques have been designed to improve a homogeneity of laser plasma heating. All of them involve a random phase plate (RPP) which breaks the laser beam into many beamlets adding a random phase shift to each of them. The beamlet interference in the laser focal spot creates a complicated pattern which can be considered as an ensemble of statistically independent speckles. Although an average over many speckles laser intensity could be very uniform, the magnitude of a local electromagnetic field in a speckle is significantly larger than the average value.

If the laser light power in a speckle is above the critical power for the selffocusing, the light is trapped in a speckle and a nonlinear laser filament is formed, which involves a plasma density depression filled with the electromagnetic field propagating along the filament axis. It is a stationary structure because the plasma pressure outside the filament is balanced by the ponderomotive force from inside. These stationary light filaments were found to be unstable with respect to the modulational instability. Modulational instability usually involves a slow growth and evolution on the ion acoustic scale. However, numerical simulations often demonstrate a very fast filament disruption which cannot be related to the modulational instability (e.g. [19]). Also the transmitted light demonstrates a red spectral shift suggesting that the near-forward stimulated Brillouin scattering plays a a significant role in the filament dynamics.

We have studied for the first time the filament instability which consistently accounts for ion acoustic dynamics in a broader range of parameters. Namely, the perturbations with a parallel wavenumber comparable to the filament phase modulation wavenumber are considered and a new resonance instability is found. It corresponds to a stimulated scattering of the fundamental waveguide mode in the filament on the resonant density perturbations into another excited eigenstate of the waveguide and could be considered as a forward SBS of a localized electromagnetic beam. Contrary to the conventional forward SBS of a wide laser beam which generates a broad spectrum of scattered wave the resonant instability in the filament has a well defined narrow maximum in the growth rate. This is a charactristic 
feature which may help to identify this instability in numerical simulations and in experiments.

\section{III.C. Thomson scattering from collisional plasmas.}

Thomson scattering (TS) cross-section in dense, high-Z plasmas depends on particle collisions. We have involved collisional effects including nonlocal transport processes in the calculations of the dynamical form factor [20]. These theories have been successfuly applied in the interpretation of TS gold disk laser plasma experiments stimulating fundamental changes in atomic physics modeling of such plasmas [21].

Building on these results we have analytically constructed an electron distribution function in the presence of a temperature gradient and the electron heat flow. Our results are obtained from the solution to the linearized Fokker-Planck equation and are valid in the entire range of particle collisionality. We have used the perturbed distribution function in the calculation of a dynamical form factor and TS cross section. As a result of the heat flow in the background plasma our solution describes an effect of the return current of cold electrons and gives rise to asymmetry of the ion acoustic peaks in the dynamical form factor. The magnitude and width of these resonances is sensitive to the nonlocal transport coefficients therefore allowing direct comparisons with different transport models. We have also examined a threshold of the return current instability in a weakly collisional regime. This instability is an important mechanism responsible for the high level of ion acoustic fluctuations in a laser produced plasmas.

\section{Laser propagation in underdense plasmas}

In this section, we discuss the results of hydrodynamic modeling of an adiabatically rising pulse through an underdense plasma which show that an evacuated channel is formed without filamentation. If the pulse is ramped up correctly, then the filamentation threshold is kept above the laser intensity because the density in the channel drops sufficiently fast. This effect of pulse shaping is supported by laser-plasma experiments which compare the propagation of pulses with square and Gaussian shapes through an underdense plasma. A larger fraction of the light stays in the original focusing cone angle when a Gaussian pulse shape is used, then when it is square, which can be interpreted as being due to reduced filamentation. 
The propagation of an intense laser pulse through a fully ionized plasma is a topic of considerable interest. When the laser intensity is sufficiently large, ponderomotive and thermal forces can modify the background plasma distribution leading to whole beam self-focusing and filamentation which can change the propagation of the laser light as well as modify the conditions under which plasma instabilities can grow. The propagation of the laser pulse to an intense focus in a plasma has specific applications in inertial confinement fusion (ICF) in the cases of hohlraum entrance holes and the fast ignitor concept [22]. There are also applications to table top Xray lasers and laser-plasma based particle accelerators. Substantial effort has gone into models of laser beams propagating through large, high density plasma, but to date quantitative comparison has been made only to experiments with pulse lengths of 100 ps [23]. Important experimental studies have been made in which a neutral gas was ionized to prepare a channel for a second pulse [24]. When one starts with a high density, fully ionized plasma, however, the filamentation instability can interfere with the formation of a density channel. In previous work we have shown that a plasma density channel is formed by focused 100 ps laser pulses in the intensity range $1 \times 10^{15}$ to $5 \times 10^{16} \mathrm{~W} / \mathrm{cm}^{2}$ and plasma densities greater than $0.1 n_{c}$. In general, the channel does not go through the plasma but has a finite axial extent due to the onset of the filamentation instability which sprays out the laser light; this requires that the laser intensity is below the filamentation threshold, a case which is not of interest to the fast ignitor application.

Simulations using the hydrodynamic simulation code LASNEX [24] show that the channel formation is affected by the laser pulse shape. For the propagation of the electromagnetic waves, LASNEX uses a ray trace/geometrical optics approximation. Modeling has been done for lasers in the intensity range of $10^{15}<\mathrm{I}<10^{17} \mathrm{~W} / \mathrm{cm}^{2}$. In this range, $\mathrm{v}_{\mathrm{o}} / \mathrm{v}_{\mathrm{e}}$, the ratio of oscillatory to electron thermal velocity, approaches or exceeds unity. Collisional effects become weak at high $\mathrm{v}_{\mathrm{o}} / \mathrm{v}_{\mathrm{e}}$ so ponderomotive filamentation / self-focusing is expected to dominate thermal effects at high intensity. Both codes included ponderomtive filamentation, but $T_{e}=T_{i}=$ constant, i.e. no thermal filamentation.

We conclude with a simulation of a laser pulse with an exponentially rising intensity starting at $1.5 \times 10^{16} \mathrm{~W} / \mathrm{cm}^{2}$, and going to $5 \times 10^{17} \mathrm{~W} / \mathrm{cm}^{2}$ with a 100 ps time constant (see Fig. 8). Figure 9 shows the transverse density profile modified by the laser beam at three different axial positions at 350 
ps. , and the plasma density at a time 350 ps. Notice that the plasma density has decreased in the channel by three orders of magnitude, so most of the pulse energy can propagate through the plasma without spraying, as shown in the ray paths shown in Figure 10. The reason for this is that the leading edge of the pulse is intense enough to form a density channel but not

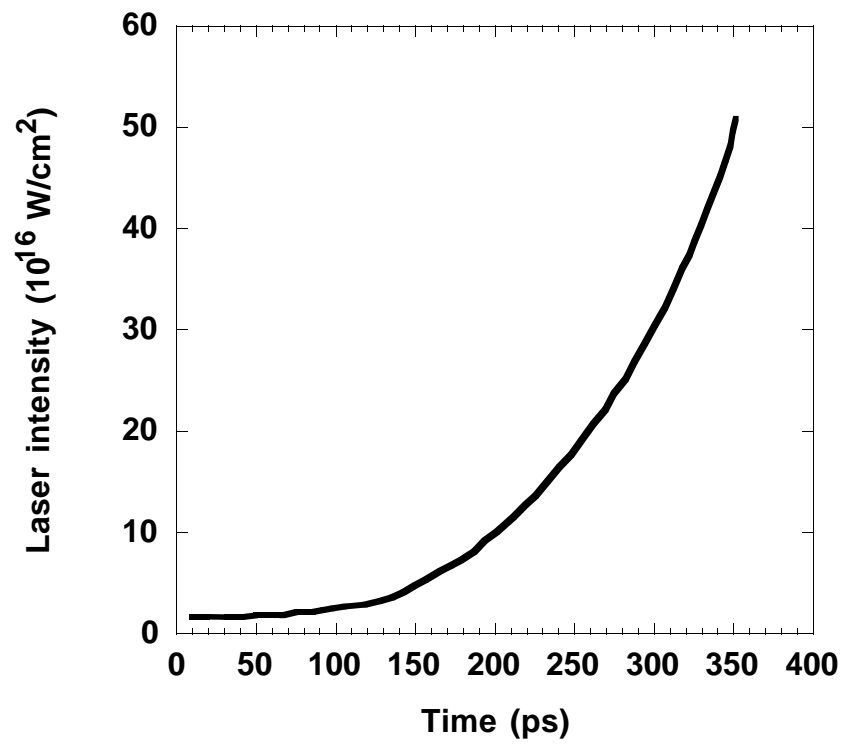

Figure 8. Time profile of the laser pulse used in the LASNEX simulation.

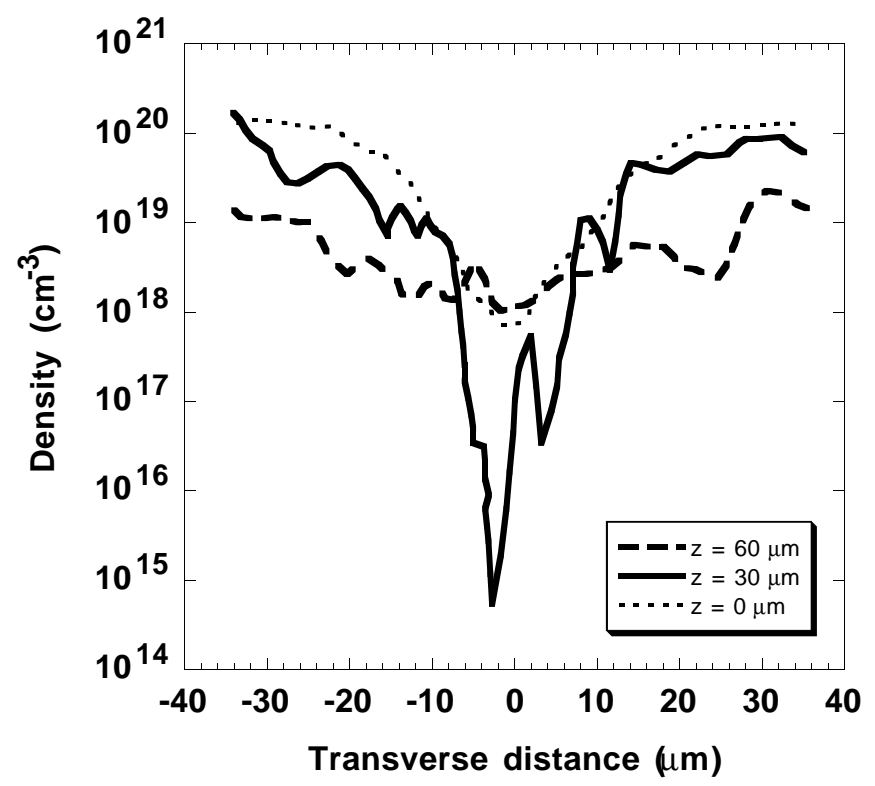

Figure 9. Transverse density profiles for three axial positions at $t=350$ ps. 


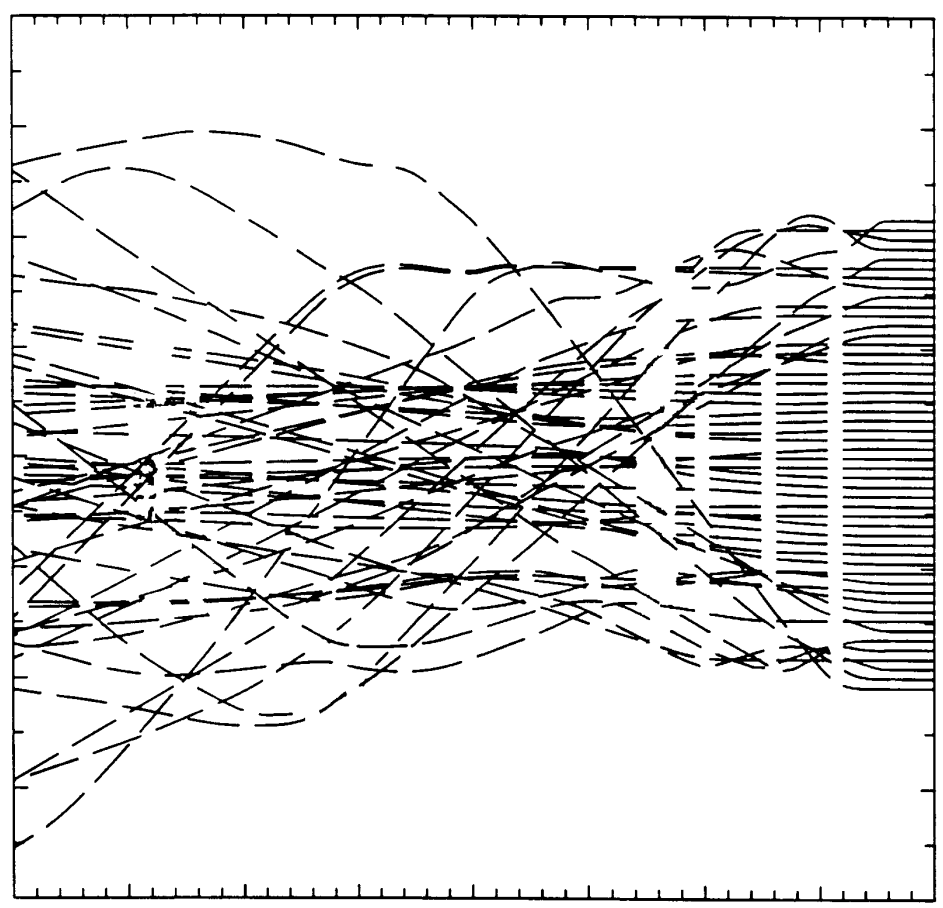

Figure 10. LASNEX rays at $\mathrm{t}=350 \mathrm{ps}$. The laser is propagating from right to left. The box is $80 \mathrm{~mm}$ high and $500 \mathrm{~mm}$ long.

sufficiently strong to filament; the density in the channel remains below the filamentation threshold throughout the pulse.This effect is seen in experiments in which either temporally square or Gaussian pulses are propagated through underdense plasmas. The gaussian-shaped pulses show a higher percentage of transmitted light in the vacuum light cone compared to square pulses.

The experiments were performed with the Janus laser at Lawrence Livermore National Laboratory. The experiments used two opposing beams, one of which was of wavelength $0.53 \mu \mathrm{m}$ that was used to explode a parylene $\left[(\mathrm{CH})_{\mathrm{n}}\right]$ foil, and the second of was of wavelength $1.064 \mu \mathrm{m}$ that provided an underdense plasma for the second, 500 ps Gaussian beam to propagate through. A $0.35-\mu \mathrm{m}$ wavelength, 50 ps probe was propagated through the target plane, perpendicular to the high energy beams, and was used for interferometry.

We can control the peak density by varying the relative timing of the plasma formation beam and the propagation beam. This affects the details of the channel profile and, if the peak density is low enough, the channel continues through to the opposing side of the plasma profile. 
As we have shown in earlier work [22,25], the main effect of filamentation and the resulting beam spray is to scatter laser light out of the vacuum solid angle. The scattering can be detected directly using filtered, infra-readsensitive film to detect the forward-scattered light, or by measuring the amount of energy collected by the opposing heater beam focusing lens (both the interaction beam and the plasma forming beam use identical $\mathrm{f} / 2$ lenses).

Comparison of interaction beams using 1-ns FWHM square and Gaussian pulses shows a $30 \%$ higher transmission for Gaussian pulses than for square pulses with the same peak intensity. This shows that pulse shaping can be a means for delivering focused laser energy through a coronal plasma to high densities.

\section{Summary}

In this project we addressed three aspects of laser-target coupling to plasma densities near the critical density, $\mathrm{n}_{\mathrm{c}}$, where the plasma frequency equals the laser frequency: (1) absorption mechanisms in high intensity, short pulse laser-target interactions with solid targets, (2) control of laser propagation properties in underdense plasmas by changing the laser pulse shape, and (3) development of simulations to accurately model the propagation of high intensity laser pulses through high density plasmas. As a result of this work we have identified important absorption mechanism at critical density, and continued to develop new modeling capabilities for designing pulse shaping to allow propagation of focused laser beams through coronal plasmas.

\section{Acknowledgements}

This work was performed under the auspices of the U. S. Department of Energy by the Lawrence Livermore National Laboratory under Contract No. W-7405-Eng-48.

\section{References}

[1] C. T. Hansen, S. C.Wilks, and P. E. Young, Phys. Rev. Lett. 83, 5019 (1999). 
[2] A. V. Maximov, R. M. Oppitz, W. Rozmus, and V.T. Tikhonchuk, Phys. Plasmas 7, 4227 (2000).

[3] V. L. Ginsburg, Propagation of Electromagnetic Waves in Plasmas, (New York, Gordon and Breach, 1960); N. G. Denisov, Sov. Phys. JETP 4, 544 (1957); D. Forslund, et al., Phys. Rev. A 11, 679 (1975).

[4] R. Sigel, J. Phys. (Paris) C6, 35 (1977).

[5] D. von der Linde et al., IEEE J. Quant. Electr. 28, 2388 (1992); in Laser

Interactions with Atoms, Solids and Plasmas, edited by R.M. More (Plenum Press, New York, 1994), p. 207.

[6] T. Engers et al., Phys. Rev. A 43, 4564 (1991); L. A. Gizzi et al., Phys. Rev. Lett. 76, 2278 (1996).

[7] W. Rozmus, et al., Phys. Plasmas 3, 360 (1996).

[8] W. Rozmus and V. T. Tikhonchuk, Phys. Rev. A 42, 7401 (1990).

[9] S. C. WIlks and W. L. Kruer, IEEE J. Quant. Elect. 33, 1 (1997); P.

Gibbon and E. Förster, Plasma Phys. Control. Fusion 38, 769 (1996).

[10] D. F. Price et al., Phys. Rev. Lett. 75, 252 (1995).

[11] R. Fedosejevs et al., Phys. Rev. Lett. 64, 1250 (1990).

[12] H. M. Milchberg and R. R. Freeman, J. Opt. Soc. Am. B 6, 1351

(1989); Phys. Rev. A 41, 2211 (1990).

[13] O. L. Landon and W. E. Alley, Phys. Rev. A 46, 5089 (1992).

[14] T. Y. B. Yang et al., Phys. Plasmas 3, 2702 (1996).

[15] F. Brunel, Phys. Rev. Lett. 59, 52 (1987).

[16] A. B. Langdon and B. F. Lasinski, Methods in Computational Physics, Vol.16, J. Killeen et al., Eds. (Academic Press, New York, NY 1976)

[17] V. Yu. Bychenkov, W. Rozmus, V. T. Tikhonchuk, and A. V. Brantov, Phys. Rev. Lett. 75, 4405 (1995); A. V. Brantov, V. Yu. Bychenkov, W.

Rozmus, and V. T. Tikhonchuk, JETP 83, 716 (1996); A. V. Brantov, V.

Yu. Bychenkov, V. T. Tikhonchuk, and W. Rozmus, Phys. Plasmas 5, 2742 (1998).

[18] V. V. Eliseev, D. Pesme, W. Rozmus, V. T. Tikhonchuk, and C. E. Capjack, Phys. Scripta T75, 112 (1998).

[19] J. Myatt, W. Rozmus, V. Yu. Bychenkov and V. T. Tikhonchuk, Phys. Rev. E 57, 3383 (1998).

[20] S. H. Glenzer, W. Rozmus, B. J. MacGowan, K. G. Estabrook, J. D. De Groot, G. B. Zimmerman, H. A. Baldis, J. A. Harte, R. W. Lee, E. A.

Williams, and B. G. Wilson, Phys. Rev. Lett. 82, 97 (1999).

[21] M. Tabak, J. Hammer, M. E. Glinsky, W. L. Kruer, S. C. Wilks, J. Woodworth, E. M. Campbell, and M. D. Perry, Phys. Plasmas 1, 1626 (1994). 
[22] S. C. Wilks, P. E. Young, J. Hammer, M. Tabak, and W. L. Kruer, Phys. Rev. Lett. 73, 2994 (1994).

[23] C. G. Durfee and H. M. Milchberg, Phys. Rev. Lett. 71, 2409 (1994).

[24] G. D. Zimmerman and W. L. Kruer, Comments Plasma Phys.

Controlled Fusion 2, 51 (1977).

[25] P.E. Young, Phys. Plasmas 2, 2815 (1995). 